Article

\title{
Unique Physical Signature of DNA Curvature and Its Implications for Structure and Dynamics \\ Dietmar Porschke
}

J. Phys. Chem. B, 2007, 111 (41), 12004-12011 • DOI: 10.1021/jp073965e • Publication Date (Web): 21 September 2007

Downloaded from http://pubs.acs.org on March 23, 2009

\section{More About This Article}

Additional resources and features associated with this article are available within the HTML version:

- $\quad$ Supporting Information

- $\quad$ Links to the 1 articles that cite this article, as of the time of this article download

- $\quad$ Access to high resolution figures

- $\quad$ Links to articles and content related to this article

- Copyright permission to reproduce figures and/or text from this article

View the Full Text HTML 


\title{
Unique Physical Signature of DNA Curvature and Its Implications for Structure and Dynamics
}

\author{
Dietmar Porschke \\ Max Planck Institut für biophysikalische Chemie, AG Biomolecular Dynamics, 37077 Göttingen, Germany
}

Received: May 22, 2007; In Final Form: August 3, 2007

\begin{abstract}
A particularly sensitive birefringence technique is used to analyze a curved DNA fragment with 118 bp and a standard DNA with $119 \mathrm{bp}$. At salt concentrations from 0.5 to $10 \mathrm{mM}$, both fragments show the usual negative stationary birefringence and monotonic transients-differences are relatively small. At $100 \mathrm{mM}$ salt the curved DNA shows a positive stationary birefringence and non-monotonic transients with processes having amplitudes of opposite sign, whereas signals of the standard DNA remain as usual. Transients induced by reversal of the field vector indicate the existence of a permanent dipole for the curved DNA. 2-MHz-ac pulses induce a negative stationary birefringence in both DNAs. These results are consistent with calculations on models for curved DNA predicting a quasi-permanent dipole and a positive dichroism/birefringence. The quasi-permanent dipole results from the loss of symmetry in the charge distribution of the curved polyelectrolyte. The appearance of the unique signature of curvature at high salt is mainly due to a strong decrease of the polarizability by about 2 orders of magnitude. The special mode of orientation resulting from the quasipermanent dipole is expected to contribute to the gel migration anomaly. The time constants of birefringence decay for the curved fragment are shorter than those of the $119 \mathrm{bp}$ fragment by a factor of $\sim 1.10$ at $0.6 \mathrm{mM}$ salt, whereas this factor is $\sim 1.20$ at $100 \mathrm{mM} \mathrm{Na}^{+}$. If both fragments were normal DNA with $3.4 \AA$ rise per base pair, the factor would be $\sim 1.02$. At high salt and high electric field strengths the factor increases up to 1.37. The implications for the bending dynamics and the potential to distinguish static from dynamic persistence by field reversal experiments are discussed. The dependence of the curvature on the salt concentration indicated by the time constants is consistent with a clear decrease of the electrophoretic anomaly at decreasing salt concentration.
\end{abstract}

\section{Introduction}

The structure of curved DNA has been studied by many different methods. ${ }^{1-8}$ Most of the available information was obtained by studies of gel mobility ${ }^{3}$ and of circularization probabilities. ${ }^{4,5}$ Obviously these techniques have been very useful, but questions on the degree of DNA curvature remained. The angle of the bend caused by sequences of four to six adenine residues has been "measured by numerous methods with results that vary by several fold". ${ }^{8}$ The predictions by currently available models for DNA curvature ${ }^{9-11}$ also show a wide variation (cf. the example presented under Results). Thus, a more direct approach by a sensitive physical technique is needed. The main problem is the fact that DNA curvature results from relatively small effects on the level of local structure, which are added up to large effects on the level of global structure. Although local structure can be determined at a high accuracy by NMR techniques, ${ }^{6}$ the accuracy is not high enough for a sufficiently accurate prediction of global structures. Among the techniques for analysis of global structures, FRET is currently the most popular, ${ }^{7}$ but is not of optimal accuracy at the long distances involved in DNA curvature. Is there any alternative?

A highly sensitive approach for analysis of global structures is electrooptical measurements, ${ }^{12,13}$ which can be used to determine changes of DNA helix lengths at the level of, e.g., 100 base pairs at a resolution of 1 base pair. ${ }^{14,15}$ This approach has also been used for analysis of DNA curvature, ${ }^{16-21}$ but the studies were restricted to very low salt concentration in most investigations. Because DNA is a polyelectrolyte, a strong influence of the ionic environment must be expected. A technique for measurements of the electric dichroism at physiological salt concentration has been developed ${ }^{22}$ and used for studies of DNA structure, ${ }^{23,24}$ but this technique requires rather high electric field strengths. Further investment into refinement of electrooptical techniques has been successful: measurements are now possible at high salt concentrations and at low electric field strengths. The results obtained by this technique provide new quantitative information about the structure and dynamics of curved DNA.

\section{Experimental Methods}

The curved DNA fragment was synthesized, cloned into the plasmid pUC20 and the sequence controlled by sequence analysis. The fragment with 118 base pairs (bp) was prepared from the plasmid DNA by the restriction nuclease SmaI, providing a fragment with the sequence GGGGATCCTC ATGTTTGACA GCTTATCATC GATATATTTT TTAGAGATTT TTATATATTT TTTAGAGATT TTTATATATT TTTTAGAGAT TTTTATATAT TTTTTAGAGA CGATACCC. This fragment was separated from the main part of the plasmid DNA by a Sephacryl S500 column and was purified further via gel electrophoresis. The fragment was dialyzed extensively first against high salt $(1 \mathrm{M} \mathrm{NaCl}, 10 \mathrm{mM}$ cacodylate $\mathrm{pH}$ 7, $1 \mathrm{mM}$ EDTA) and finally against $1 \mathrm{mM} \mathrm{NaCl}, 1 \mathrm{mM}$ cacodylate $\mathrm{pH} 7$.

The reference fragment with 119 bp was cut from the same plasmid by the restriction nucleases $P d m \mathrm{I}$ and $R s a \mathrm{I}$, providing 
the sequence TTTTCCAATG ATGAGCACTT TTAAAGTTCT GCTATGTGGC GCGGTATTAT CCCGTATTGA CGCCGGGCAA GAGCAACTCG GTCGCCGCAT ACACTATTCT CAGAATGACT TGGTTGAGT. This fragment was isolated by the procedures described above.

The electrophoretic mobility was measured in a thermostated gel holder in TBE buffer containing $89 \mathrm{mM}$ Tris/boric acid $\mathrm{pH}$ 8.1, $2.5 \mathrm{mM}$ EDTA and in diluted versions of this buffer. As a reference a 100 bp ladder "O'RangeRuler 100bp" from Fermentas was used; the gels were scanned and evaluated using the software Quantity One from Bio-Rad.

Solutions of the fragments were prepared for measurements in the following buffers: $\mathrm{E}: 1 \mathrm{mM} \mathrm{NaCl}, 1 \mathrm{mM}$ cacodylate pH 7.0, 0.2 mM EDTA; M: $1 \mathrm{mM} \mathrm{NaCl}, 1 \mathrm{mM}$ cacodylate $\mathrm{pH}$ 7.0, $100 \mu \mathrm{M} \mathrm{MgCl}_{2}$; the buffers $\mathrm{E}$ and $\mathrm{M}$ were also used diluted by a factor of 4, resulting in $\mathrm{E} / 4$ and $\mathrm{M} / 4$, respectively; E10: $11 \mathrm{mM} \mathrm{NaCl}, 1 \mathrm{mM}$ cacodylate $\mathrm{pH}$ 7, 0.2 mM EDTA; M10: $10 \mathrm{mM} \mathrm{NaCl}, 1 \mathrm{mM}$ cacodylate pH 7.0, $0.2 \mathrm{mM} \mathrm{MgCl}_{2}$; EH: $70 \mathrm{mM} \mathrm{NaCl}, 35 \mathrm{mM}$ cacodylate pH 7.0, 0.2 mM EDTA; $\mathrm{MH}$ : $70 \mathrm{mM} \mathrm{NaCl}, 35 \mathrm{mM}$ cacodylate $\mathrm{pH} 7.0,1 \mathrm{mM} \mathrm{MgCl}_{2}$. The cacodylate buffers were prepared from cacodylic acid and the $\mathrm{pH}$ was adjusted by addition of $\mathrm{NaOH}$; thus, the $\mathrm{Na}^{+}$concentration of buffers $\mathrm{EH}$ and $\mathrm{MH}$ was $100 \mathrm{mM}$.

The general scheme of birefringence setup corresponds to the "classical" one described by Fredericq and Houssier. ${ }^{12}$ However, the sensitivity has been extended by a novel construction of the cell, avoiding strain in the cell windows as much as possible. Furthermore, the sensitivity was increased by using stable lasers of high intensity. The highest intensity used in the present investigation was $5 \mathrm{~W} \mathrm{cw}$. Finally, the optics was optimized by using components of high quality. Direct current (dc) pulses were generated by Cober Model 606 pulse generators. The polarity was inverted after each pulse application automatically. Alternating current (ac) pulses were generated by an arbitrary function generator AFG 3101 from Tektronix together with a linear pulse power amplifier LPPA 6020 from dressler HF-Technik GmbH (Stolberg, Germany). The optical path length of the cell used in the present investigation was 20 $\mathrm{mm}$; the distance between the platinum electrodes was $2.68 \mathrm{~mm}$. The response time of the detector used for most measurements was $100 \mathrm{~ns}$. The transients shown in the present investigation represent the average resulting from 20 pulse applications. The relative change of light intensities is given in voltage units [V] as measured by the detector. For calculation of the birefringence and of the Kerr constants, these units were converted using the equations described by Fredericq and Houssier. ${ }^{12} \mathrm{~A}$ publication with more technical details about the instrument and its performance is in preparation. All birefringence data presented in this paper were measured in the limit of low concentrations $c$ and/or electric fields strengths $E$, where the time constants did not show any dependence on $c$ or $E$ and the amplitudes were in the Kerr law range. Typical values of $c$ and $E$ are given in the legends to the figures. Special conclusions resulting from studies of effects out of the Kerr law range will be reported separately. All the data obtained for DNA samples in buffers with $100 \mathrm{mM} \mathrm{Na}^{+}$were corrected for the birefringence contribution of the buffer by subtraction of birefringence transients measured for the buffer alone under identical conditions (i.e., electric field strength and pulse length).

The electric dichroism at high field strengths was measured with an instrument described previously. ${ }^{22}$ In this case the orientation effects were clearly outside the Kerr law range. All data were obtained at $2{ }^{\circ} \mathrm{C}$. Changes of time constants due to temperature jump effects were corrected by the usual viscositytemperature factor. ${ }^{23}$

Exponentials were fitted by analytical convolution equations ${ }^{25}$ representing the detector response by an exponential or by a numerical convolution procedure. ${ }^{26}$

\section{Results}

Gel Mobility and Model Predictions. In the present investigation the structure and dynamics of two different DNA fragments, one with $118 \mathrm{bp}$ and another one with $119 \mathrm{bp}$, are analyzed in solution. The fragment with 118 bp has seven A-tracts arranged with a periodicity of $10.5 \mathrm{bp}$, a sequence feature known to induce strong curvature. In agreement with this expectation the $118 \mathrm{bp}$ fragment shows a reduced gel mobility-in $5 \%$ polyacrylamide gels it migrates like a DNA double helix with $\sim 154 \mathrm{bp}$ in TBE buffer at $20{ }^{\circ} \mathrm{C}$. When the buffer concentration is reduced by a factor of 10 to an ion concentration of $\sim 4.7 \mathrm{mM}$, the $118 \mathrm{bp}$ fragment migrates like a standard DNA with $\sim 141 \mathrm{bp}$. This result shows that the migration anomaly of curved DNA decreases at low salt-in agreement with the electrooptical data described below.

Currently available models for prediction of helix structures from nucleotide sequences ${ }^{9-11}$ provide very different curved trajectories with end-to-end distances ranging from $150 \AA$ over $260 \AA$ to $330 \AA$ (the end-to-end distance of straight DNA with 118 bp is $401 \AA$ ). The gel mobility of the reference fragment with $119 \mathrm{bp}$ is normal, and the models predict a straight conformation.

Stationary Birefringence and Shape of Transients. Birefringence transients measured for the DNA fragments by the new instrument in the low salt regime are as expected from results obtained previously. Both fragments show a negative stationary birefringence, and the amplitudes in the regime of low electric fields are a linear function of the square of the field strength, consistent with the Kerr law. Due to the high sensitivity, transients can be measured at a higher signal-tonoise ratio and/or measurements can be extended to lower DNA concentrations or to lower electric field strengths. At low DNA concentrations the birefringence signal of water is of the same order of magnitude as the signal of the DNA (Figure 1). Because the time constants of these two processes are quite different, separation is relatively simple at a sufficiently high bandwidth of the detector.

When the salt concentration is increased, the amplitude of the DNA birefringence decreases, but the stationary values remain negative up to medium salt concentrations. For the curved DNA fragment the stationary birefringence is still negative in buffer M10 (ionic strength $11 \mathrm{mM}$ ). A further increase of the ionic strength to $100 \mathrm{mM}$ (buffer EH) results in a reversal of the birefringence (Figure 2). Under these conditions the birefringence amplitude of the DNA is of a magnitude similar to that of the water. For a simple identification of the relaxation components of the DNA, it is useful to subtract the birefringence response of the buffer from that of DNA dissolved in the same buffer. With the exception of the data shown in Figure 1, buffer signals were subtracted from all transients shown in this contribution (Figures 2-5). The result clearly shows that (1) the stationary birefringence of the DNA with $118 \mathrm{bp}$ in buffer EH is positive and (2) the transients are non-monotonic, because they show relaxation processes with amplitudes of opposite sign.

Under the same buffer conditions the reference DNA fragment with 119 bp shows monotonic transients with a negative stationary birefringence (Figure 3). Thus, the electrooptical 
a

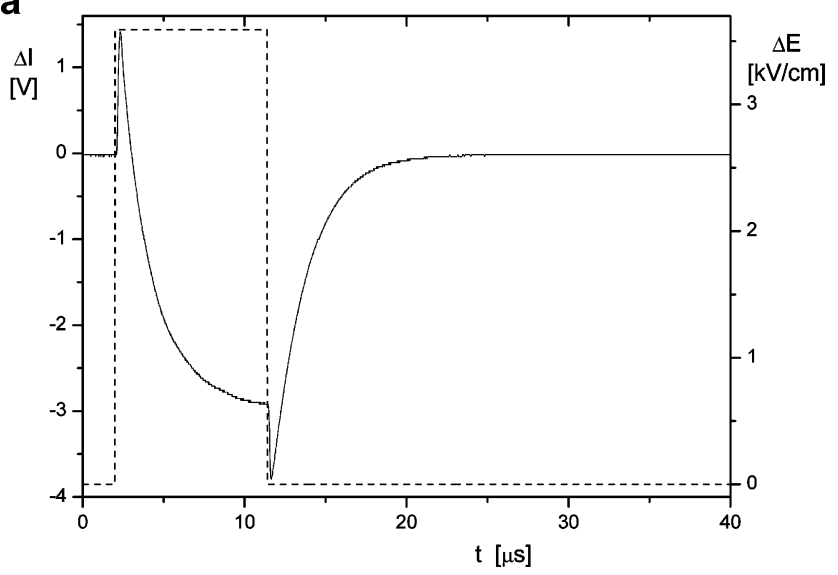

b

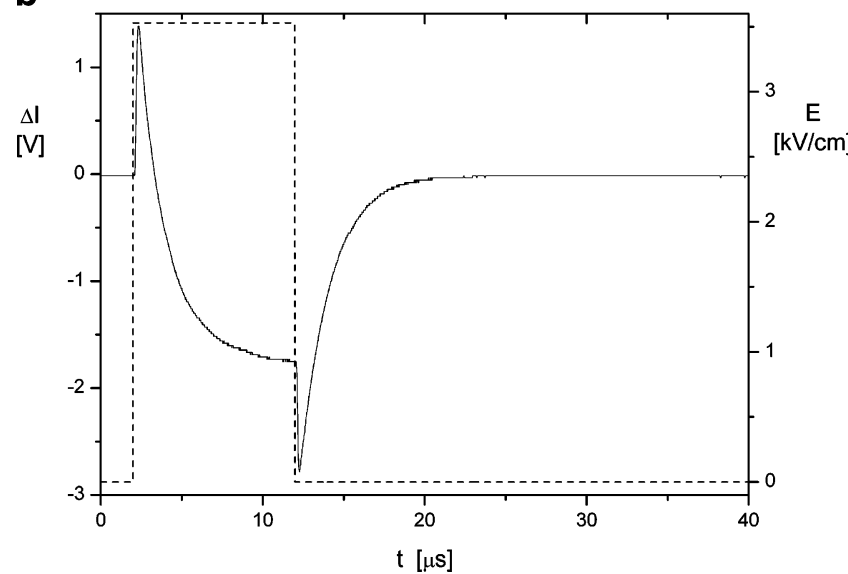

Figure 1. Change of light intensity $\Delta I$ reflecting birefringence (continuous line, left scale) as a function of time $t$ for the DNA (a) with $119 \mathrm{bp}(57.5 \mathrm{nM}$ helix) and (b) with $118 \mathrm{bp}$ (52.2 $\mathrm{nM}$ helix) measured in buffer $\mathrm{M}$ at $2{ }^{\circ} \mathrm{C}$. The electric field pulse $\Delta E$ (right scale) is shown by the dashed line. The birefringence transients are not corrected for the contribution of the buffer. Thus, the first parts of the rise and of the decay curves reflect the birefringence of water (time constants shorter than that of the detector). The second, slow parts of the transients reflect the birefringence of the DNA (time constants see Table 1).

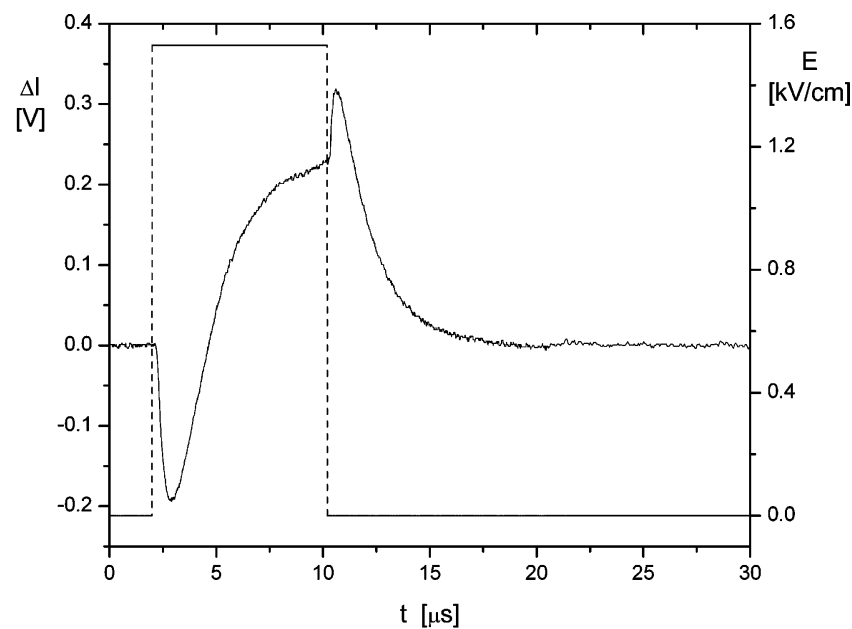

Figure 2. Change of light intensity $\Delta I$ reflecting birefringence (continuous line, left scale) as a function of time $t$ for the DNA with $118 \mathrm{bp}$ measured in buffer $\mathrm{EH}\left(2{ }^{\circ} \mathrm{C}\right.$, DNA helix concentration 782 $\mathrm{nM}$ ). The electric field pulse $\Delta E$ (right scale) is shown by the dashed line. The birefringence of the buffer has been subtracted.

results obtained for the curved and the standard DNA fragments are very different. The nature of this difference can be analyzed

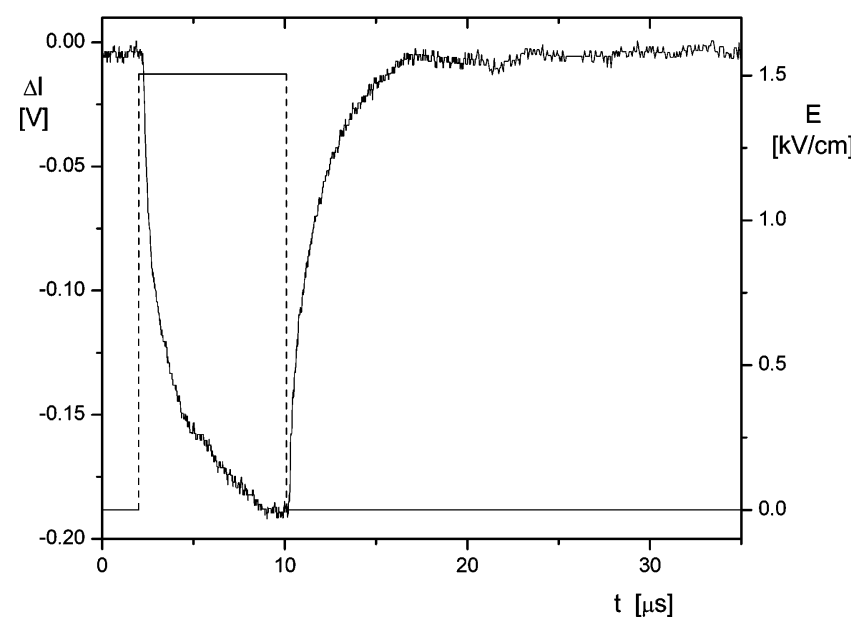

Figure 3. Change of light intensity $\Delta I$ reflecting birefringence (continuous line, left scale) as a function of time $t$ for the $119 \mathrm{bp}$ DNA in buffer $\mathrm{EH}\left(2{ }^{\circ} \mathrm{C}\right.$, DNA helix concentration $\left.323 \mathrm{nM}\right)$. The electric field pulse $\Delta E$ (right scale) is shown by the dashed line. The birefringence of the buffer has been subtracted.

by application of sine pulses with sufficiently high frequency. As shown in Figure 4, the stationary birefringence of the curved DNA fragment is reverted to a negative one in electric fields with a high frequency of $2 \mathrm{MHz}$. This result indicates that the orientation in dc electric fields is dominated by a permanent dipole moment, which is perpendicular to the eigenvector of the largest eigenvalue of the polarizability tensor. Alternatively, a slow polarizability would have to be postulated with a direction perpendicular to that of the fast polarizability. As will be shown in the Discussion, a reasonable physical basis is known for the first interpretation but not for the second one.

The line of arguments is complemented by the fact that the stationary birefringence of the reference fragment with $119 \mathrm{bp}$ at $100 \mathrm{mM} \mathrm{Na}^{+}$is negative in both dc and high-frequency ac electric fields. Finally, the conclusion on the existence of a permanent dipole in the curved DNA fragment is supported by the transient observed upon reversal of the electric field vector (Figure 5). The dip in the birefringence induced by reversal of the field vector ${ }^{27}$ reflects turning of the preferential direction of permanent dipoles by $\sim 180^{\circ}$. In the case of induced dipoles with a fast polarizability, the direction of the dipole is reverted quickly without rotation of the molecules and, thus, a dip does not appear. Birefringence signals without the dip upon reversal of the field vector were observed for the fragment with $119 \mathrm{bp}$ (data not shown). The large dip upon reversal of the field vector was also not observed, when the 118 bp fragment was studied in the buffers with lower salt concentrations.

Decay Time Constants in Different Buffers. The decay curves measured after pulse termination provide detailed information about the hydrodynamic dimensions and, thus, were analyzed over a wide range of buffer conditions. As a test for potential aggregation effects, the decay curves were measured at different DNA concentrations. These data (not shown) demonstrate that the time constants are independent of the DNA concentration in the range below $\sim 1 \mu \mathrm{M}$ DNA helices, whereas higher DNA concentrations lead to an increase of the time constants. A compilation of time constants measured in the limit of low DNA concentrations is given in Table 1.

Dichroism Measurements at High Electric Field Strength. The new birefringence technique was used at relatively low electric field strengths, because the currently available pulse generator did not have enough power for higher voltages at given resistances. For a comparison with data at high field 


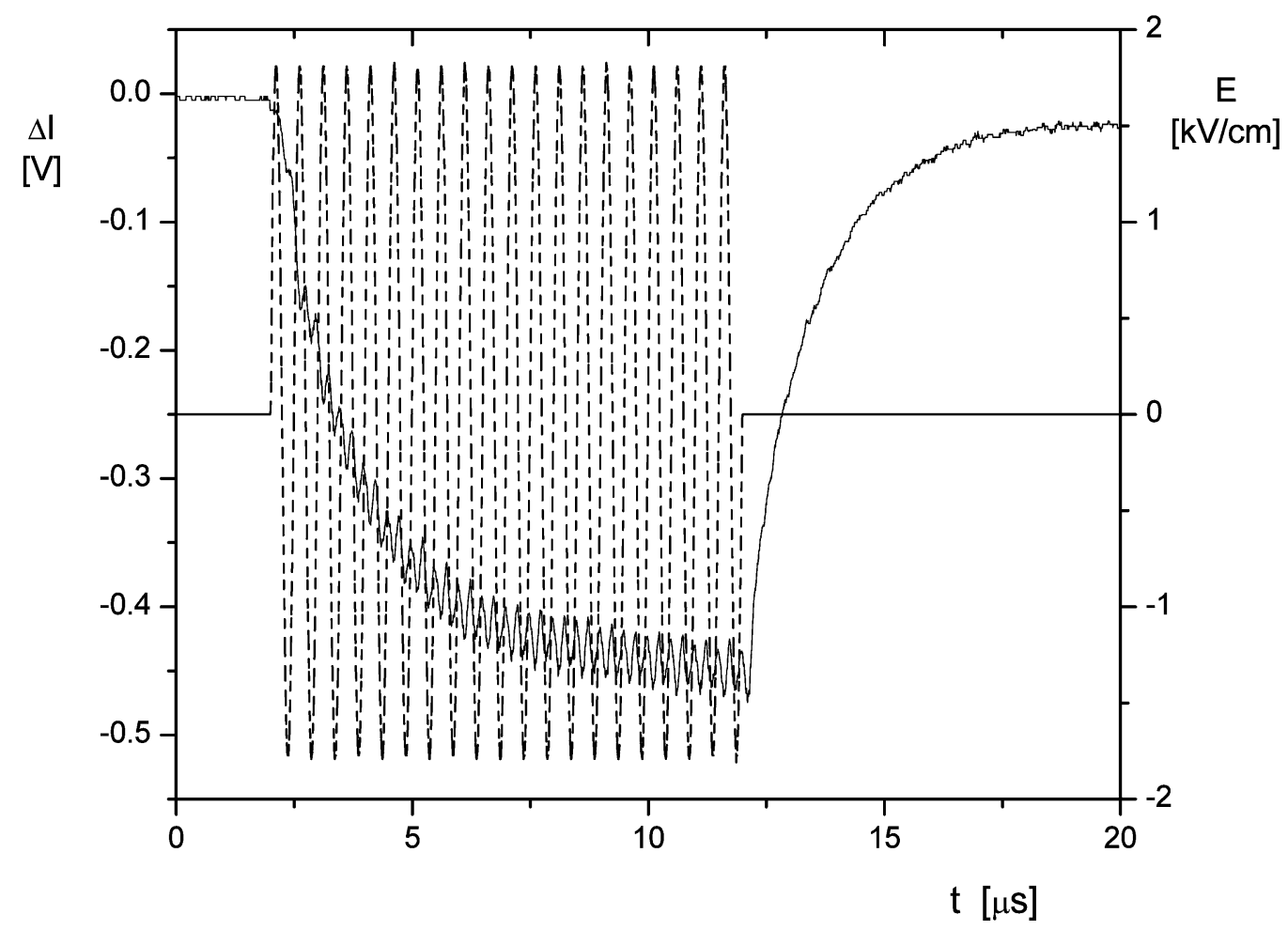

Figure 4. Change of light intensity $\Delta I$ reflecting birefringence (continuous line, left scale) as a function of time $t$ for the DNA with 118 bp in buffer $\mathrm{EH}$ induced by an ac pulse of $2 \mathrm{MHz}\left(2{ }^{\circ} \mathrm{C}\right.$, DNA helix concentration $684 \mathrm{nM}$ ). The electric field pulse $\Delta E$ (right scale) is shown by the dashed line. The birefringence of the buffer has been subtracted.

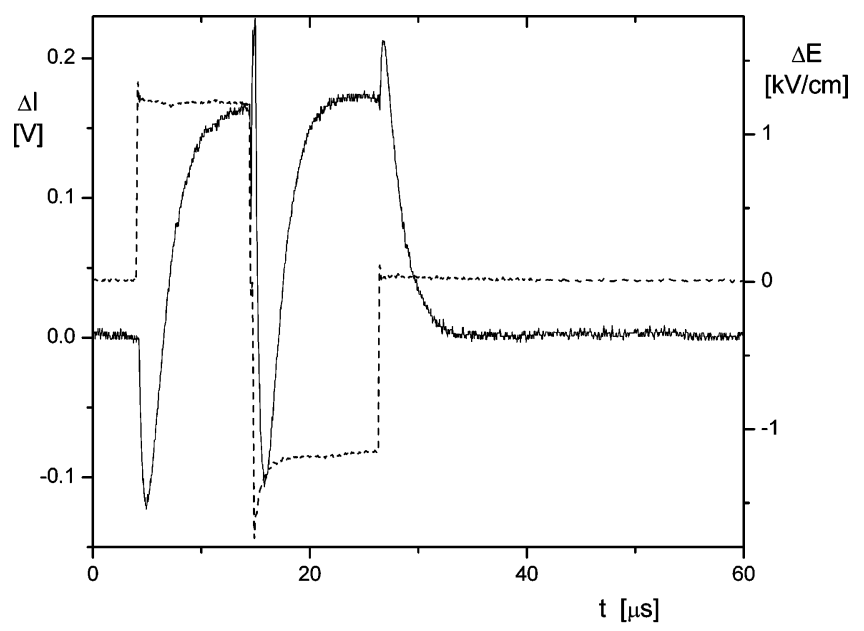

Figure 5. Change of light intensity $\Delta I$ reflecting birefringence (continuous line, left scale) as a function of time $t$ for the $118 \mathrm{bp} \mathrm{DNA}$ in buffer $\mathrm{EH}$ induced by a dc pulse with reversal for the polarity $\left(2{ }^{\circ} \mathrm{C}\right.$, DNA helix concentration $\left.782 \mathrm{nM}\right)$. The electric field pulse $\Delta E$ (right scale) is shown by the dashed line. The birefringence of the buffer has been subtracted.

strengths, the DNA samples were also analyzed by a previously developed technique that can be used to measure the electric dichroism at physiological salt concentrations by application of short pulses of high field strengths. ${ }^{22}$ The electric dichroism was measured in the range of electric field strengths from $\sim 40$ to $\sim 90 \mathrm{kV} / \mathrm{cm}$, and thus, the data were obtained in a range of the orientation function far above that covered by the birefringence measurements. This is reflected by the fact that the electric dichroism of both fragments was negative. It should be mentioned here that the signs of birefringence and dichroism under given experimental conditions are equivalent, and thus, a negative birefringence corresponds to a negative dichroism. The change of the sign in the case of the curved DNA fragment upon increase of the field strength reflects a turn of the average orientation mode. This turn is expected, because induced dipoles become dominant over permanent dipoles at high field strengths. The effect results from the quadratic increase of the energy for induced dipoles compared to the linear increase for permanent dipoles.

The time constants obtained from the dichroism decay at high field strengths are clearly smaller than those derived from the birefringence decay at low field strengths (in the buffers EH and $\mathrm{MH}$; Table 1). This effect is larger for the curved DNA than for the reference fragment. Apparently there is a distribution of different bending states and these bending states are not converted into each other with time constants shorter than the time constant of rotational diffusion.

For a further comparison the decay of the electric dichroism was also measured in the buffer M10 with an ionic strength of $\sim 11 \mathrm{mM}$. Here, the difference between the dichroism decay times at high field strengths and the birefringence decay times at low field strengths is much smaller (118 bp birefringence low field $1.74 \mu \mathrm{s}$, dichroism high field $1.65 \mu \mathrm{s} ; 119 \mathrm{bp}$ birefringence low field $1.94 \mu \mathrm{s}$, dichroism high field $1.93 \mu \mathrm{s}$ ).

Kerr Constants. The birefringence data were measured in the range of electric field strengths $E$, where the amplitudes show a linear increase with $E^{2}$, the so-called "Kerr regime". The magnitude of the amplitudes can be expressed in the form of Kerr constants, representing the amplitude at unit electric field strength and unit concentration. These Kerr constants show a strong decrease with increasing salt concentration (cf. Figure $6)$. At the standard conditions of low salt concentrations $(\leq 1 \mathrm{mM})$ used in most previous electrooptical investigations, the Kerr constants of the DNA fragments with 118 and $119 \mathrm{bp}$ are higher by almost 2 orders of magnitude than those at physiological salt concentrations. This indicates the substantial extension of the sensitivity that was required for birefringence measurements at physiological salt concentrations. 
TABLE 1: Decay Time Constants at $2{ }^{\circ} \mathrm{C}^{a}$

\begin{tabular}{|c|c|c|c|c|c|c|}
\hline \multirow[b]{2}{*}{ buffer } & \multirow[b]{2}{*}{ electric field } & \multirow[b]{2}{*}{$\mathrm{Na}^{+}[\mathrm{mM}]$} & \multirow[b]{2}{*}{$\mathrm{Mg}^{2+}[\mathrm{mM}]$} & \multicolumn{2}{|c|}{ decay time constants $(\mu \mathrm{s})$} & \multirow[b]{2}{*}{ factor } \\
\hline & & & & $118 \mathrm{bp}$ & 119 bp & \\
\hline $1 / 4 \mathrm{E}$ & low & 0.6 & 0 & 1.94 & 2.13 & 1.10 \\
\hline $\mathrm{E}$ & low & 2.4 & 0 & 1.83 & 2.10 & 1.15 \\
\hline $1 / 4 \mathrm{M}$ & low & 0.46 & 0.025 & 1.78 & 1.98 & 1.11 \\
\hline M & low & 1.84 & 0.1 & 1.70 & 1.90 & 1.12 \\
\hline M10 & low & 10.8 & 0.2 & 1.74 & 1.94 & 1.11 \\
\hline $\mathrm{EH}$ & low & 100 & 0 & 1.63 & 1.95 & 1.20 \\
\hline MH & low & 100 & 1 & 1.71 & 2.03 & 1.19 \\
\hline EH & high & 100 & 0 & 1.36 & 1.87 & 1.37 \\
\hline MH & high & 100 & 1 & 1.40 & 1.84 & 1.31 \\
\hline
\end{tabular}

${ }^{a}$ Data from birefringence at low electric field and from dichroism at high electric field. Estimated accuracy: buffers with low ion concentration up to $\mathrm{M} 10, \pm 1.5 \%$; buffers $\mathrm{EH}$ and $\mathrm{MH}, \pm 3 \%$. The factor is the ratio of the time constants for the fragments with 119 and 118 bp.

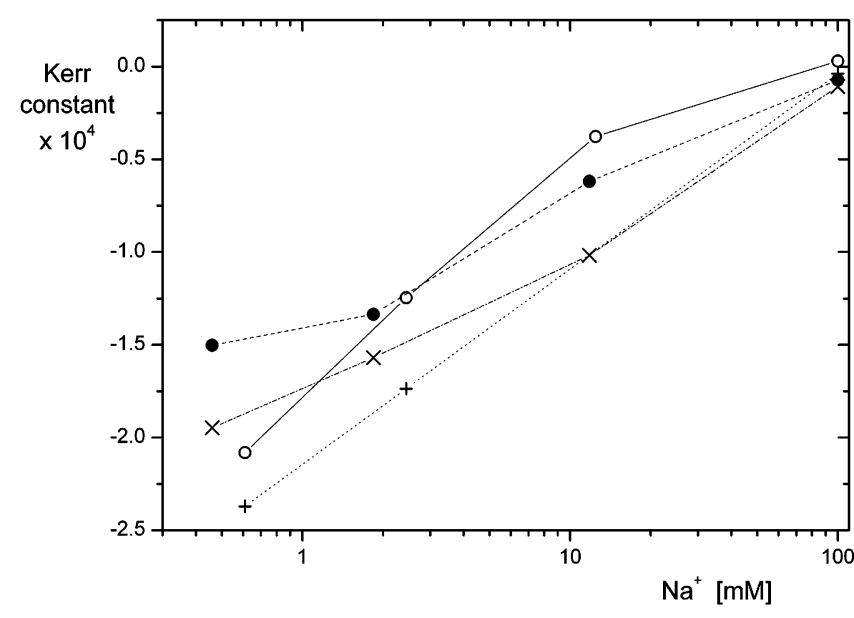

Figure 6. Kerr constants $\left[\mathrm{cm}^{2} / \mathrm{kV}^{2}\right]$ for the fragments with 118 and $119 \mathrm{bp}$ at different ion concentrations. Buffers with EDTA, $118(\mathrm{O})$ and $119 \mathrm{bp}(+)$; buffers with $\mathrm{Mg}^{2+}, 118(-)$ and $119 \mathrm{bp}(\times)\left(2{ }^{\circ} \mathrm{C}\right.$; cf. Material and Methods for the exact composition of the buffers). The contribution of the buffer has been subtracted.

\section{Discussion}

Potentials and Problems of Electrooptics. The electrooptical analysis of macromolecular structures in solution is particularly sensitive due to the strong dependence of the measured time constants on the dimensions. In addition, electrooptics offers an unusually high time resolution, providing the chance for studies of the dynamics in a time range that is hardly accessible by other techniques. In the past these advantages could not be exploited as much as necessary because of limitations to low salt concentrations. The present example demonstrates that these limitations can be removed by application of advanced techniques.

The analysis of DNA structure in solution has always been a favorite subject in electrooptics, because a particularly high accuracy is enabled by large signals resulting from the high optical and electrical anisotropy of the double helix. Another unique advantage comes from the fact that restriction fragments with exactly defined sequences and chain lengths can be prepared in quantities sufficient for electrooptical measurements. The results obtained from electrooptical investigations have been useful to characterize persistence lengths, ${ }^{23,28}$ for example.

Electrooptical procedures have also been used to characterize DNA curvature. ${ }^{17-21,24}$ The conclusions on DNA curvature derived from previous electrooptical measurements are contradictory and require some comments. First of all, DNA curvature is expected to result in reduced time constants of rotational diffusion compared to those of standard DNA of the same chain length. However, standard DNA and curved DNA are subject to thermal bending. Thus, it must be expected that the difference between standard and curved DNAs disappears at high chain lengths and that time constants of DNA samples with different degrees of curvature are no longer very different when chain lengths are in the range of a few hundred base pairs and above. This has been verified by electrooptical experiments. ${ }^{24}$ Thus, the analysis of DNA curvature should be more promising in the range of low chain lengths.

Electrooptical investigations presented in the past reported some reduction of the time constants for curved DNA compared to those for standard DNA of the same contour length. However, not all of these results can be considered as conclusive. In some cases the existence of more than a single time constant was not recognized (cf. Results section of Levene et al. ${ }^{18}$ ). Other reports claiming large effects may be due to data with a special perturbation: it is now well documented that electrooptical decay curves with artificially short time constants may result from superposition of relaxation effects with opposite amplitudes. ${ }^{13,29-31}$ Erroneous conclusions from such effects can be avoided, ${ }^{31}$ but special attention is required.

Almost all previous studies were conducted at salt concentrations of $\sim 1 \mathrm{mM}$ and even lower values. The well-known impact of electrostatics on the structure resulting from the polyelectrolyte character of DNA double helices has been neglected. The data obtained in the only previous electrooptical investigation at high salt concentrations ${ }^{24}$ were partly affected by aggregation resulting from complementary single-stranded segments at the helix ends. Moreover, efforts were concentrated on relatively long DNA fragments.

The instrument used in the present investigation has a much higher sensitivity than any instrument in the past. Thus, electrooptical parameters can be characterized at a much higher accuracy than previously under usual conditions, and furthermore, the measurements can be extended to physiological salt concentrations at low electric field strengths.

The new results confirm the conclusion that the difference in the electrooptical parameters of normal and curved DNA fragments is relatively small at low salt concentrations. ${ }^{19,24}$ The expected large difference between curved and standard DNAs is found at high salt concentrations, where electrostatic repulsion is reduced considerably. Part of the difference is apparently due to some increase of DNA curvature with increasing ionic strength. A crucial factor for the appearance of the special electrooptical effects at high salt concentrations is the strong decrease of the polarizability with increasing salt concentration. At lower salt concentration any influence of the quasi-permanent dipole moment is hidden by the dominance of the induced dipole.

Quasi-Permanent Dipole of Curved DNA. Permanent dipoles are usually not expected for molecules like DNA. Although the double helix clearly is a chiral structure, the 
distribution of charged residues is practically symmetric, as long as the double helix remains straight. However, the symmetry of the charge distribution is lost when the DNA is curved. ${ }^{29} \mathrm{~A}$ nonsymmetric distribution of charges leads to a torque in the presence of external electric fields. A dipole moment describing this torque must be referred to the center of diffusion. Calculations demonstrated that the magnitude of such dipole moments is considerable. ${ }^{29}$ These dipole moments are directed perpendicular to the end-to-end vectors of circular arc segments and, thus, explain the observation of the positive birefringence. Calculations have also been extended to the case of DNA chains with more complex shapes ${ }^{32}$ expected from the wormlike chain model. Most of the wormlike chains show permanent dipoles together with positive birefringence or dichroism values.

Experimental data showing a positive stationary dichroism together with transients indicating permanent dipoles were obtained for DNA fragments in the range of chain lengths from 399 to 859 bp at relatively low salt concentrations. ${ }^{32}$ Thus, the combination of a positive stationary dichroism with a permanent dipole is not really new for DNA double helices. However, the present results demonstrate for the first time the "unusual" effects for a relatively short DNA fragment and the correlation of these unusual effects with DNA curvature by the comparison with a standard DNA fragment. The correlation has not been found previously because previous measurements were restricted to low salt concentration, where effects resulting from high polarizabilities are dominant. The decrease of the polarizability with increasing salt concentration is demonstrated by the Kerr constant, which decreases by about 2 orders of magnitude when the salt concentration is increased from 1 to $100 \mathrm{mM}$ (cf. Figure 6 ). The magnitude of the Kerr constant is determined by the optical anisotropy and the electrical anisotropy. ${ }^{12}$ For the case of the reference fragment with $119 \mathrm{bp}$, it is likely that the contribution from the optical anisotropy is almost constant and, thus, the change is mainly due to the polarizability. The strong reduction of the polarizability at high salt concentration is essential for uncovering the quasi-permanent dipole. A decrease of the DNA polarizability with increasing salt concentration has been observed previously in other investigations, but the analysis has never been extended over such a wide range up to salt concentrations around $0.1 \mathrm{M}$.

According to the theory of electrooptics, the type of transient observed upon pulse reversal for the curved DNA may also be due to a slow polarizability (cf. refs 27 and 33). However, the slow polarization of DNA is required in a direction perpendicular to that of the end-to-end vector, which is known to have a fast polarization. ${ }^{34} \mathrm{~A}$ reasonable physical explanation for such a special combination does not exist. The high polarizability of DNA is due to a field-induced change of the ion distribution around the double helix. On the basis of the well-known rules of ion dynamics ${ }^{35}$ it remains completely unclear how the postulated slow polarizability for a DNA fragment with $118 \mathrm{bp}$ in the presence of standard monovalent ions could come about. Simulations of field-induced ion polarization around DNA double helices by Brownian dynamics ${ }^{36}$ did not provide any evidence for the existence of slow effects. In summary, a positive birefringence together with a quasi-permanent dipole moment is expected for curved DNA from elementary physics, whereas a physical basis for a slow polarizability of DNA in the presence of monovalent ions such as $\mathrm{Na}^{+}$is not known and is unlikely to exist.

The magnitude of the quasi-permanent dipole moment cannot be extracted from the present experimental data by any simple procedure, because electrooptical amplitudes for curved objects are strongly affected by coupling of translational and rotational diffusion. $^{30}$ Thus, standard orientation functions cannot be applied. However, it is clear from the results of simulations that the dipole moments resulting from the loss of symmetry are considerable. This is due to the high charge density and the large dimensions of the DNA fragments. The expected dipole moments are on the order of several thousand debye units and should have a considerable impact on molecular interactions. For example, the dipole moment of curved DNA may serve to support binding of proteins. It is known that many DNA binding proteins have a large dipole moment ${ }^{37,38}$ resulting from the asymmetry of their charge distribution. The asymmetry is mainly due to the fact that these proteins are complementary to the highly charged DNA double helix. Thus, the positive end of the protein dipole will be directed to the concave side of curved DNA fragments, representing the negative side of the curved DNA dipole. ${ }^{29}$

The quasi-permanent dipole moment induces a special orientation of curved DNA fragments in external electric fields with the end-to-end vector oriented preferentially in a direction perpendicular to the field vector. In gel matrixes this mode of orientation does not support a fast entrance of the fragments into pores of the gels. Thus, the special mode of orientation is expected to contribute to the migration anomaly in gel electrophoresis.

The new experimental technique can be used to explore the electrostatics of DNA fragments in further detail. For example, site binding of ions to specific nucleotide sequences is expected to be more important at high ion concentrations. Such effects may contribute to indirect readout and can now be analyzed by electrooptical studies of different DNA sequences.

Finally, it should be mentioned that the present discussion is restricted to the electrooptical effects resulting from DNA bending/curvature. Obviously there are other possibilities to generate long-lived anisotropic charge distributions in DNA, for example by unzippering at one of the ends or by introduction of an internal loop at one of the ends. These effects are not expected to result in the special combination of a positive birefringence/dichroism with a permanent dipole.

Static versus Dynamic Persistence. The lifetime of any given bent conformation at ambient temperatures is limited. During this lifetime its dipole persists and, thus, is "quasipermanent". However, it is subject to changes in the time range of the bending time constant. For standard DNA the different bending states result from fluctuations around the straight conformation, whereas the center of fluctuations in curved DNA is a curved state (Figure 7). In both cases the average state is bent and, thus, it may be difficult to distinguish between them. ${ }^{39}$ The present results reveal a new approach to analyze the nature of bending. This approach is dependent on the magnitude of bending time constants. For simplicity, it may be assumed first that bending is fast compared to overall rotational diffusion.

In the case of curved DNA there is a preferential direction of bending. It cannot be excluded that a curved DNA reverts its bending direction, but this will be a rare event. When curved molecules have been oriented by an external electric field due to their quasi-permanent dipole, reversal of the field vector induces rotation by $\sim 180^{\circ}$, leading to a large dip in the electrooptical transient.

In the case of standard DNA, molecules in given bending states may be oriented by an external field like a curved DNA. However, upon reversal of the field vector the molecules do not have to turn around by $\sim 180^{\circ}$. When bending is sufficiently fast, the terminal segments of the DNA may move rapidly 

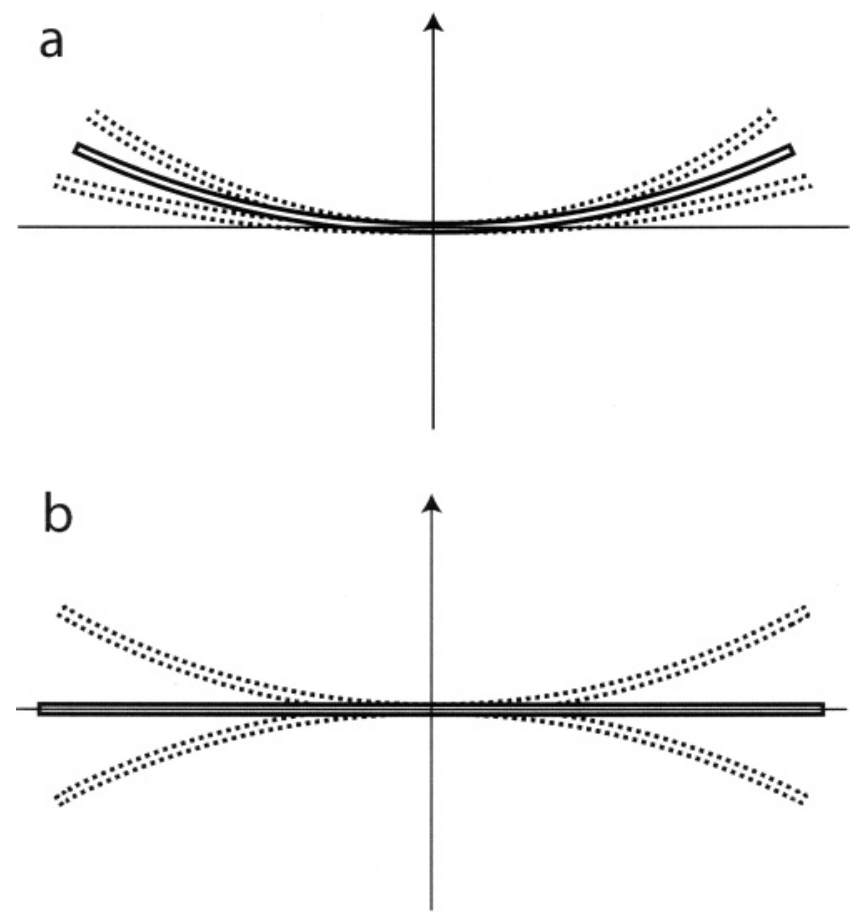

Figure 7. Scheme of bending states (a) for curved DNA and (b) for standard DNA according to a simple weakly bent rod model.

around and the opposite state of bending may be generated within a short time period. Thus, reversal of the field vector is not expected to induce a large dip in the electrooptical transient. If individual bending states exist for very short times only, then the dipole moments resulting from bending also have a very short lifetime. In this case the time required for alignment may exceed the lifetime of the dipole and the extent of field-induced alignment due to quasi-permanent dipoles may be negligible. The lifetimes of dipoles may be extended by the external electric field. Model calculations are required to assign the magnitude of this effect. In any case, transients upon reversal of the field vector are not expected to induce large dips for standard DNA with a high bending mobility.

Thus, electrooptical transients can be used to analyze the nature of DNA bending and to distinguish static from dynamic persistence. ${ }^{39}$ Obviously, the ratio of bending time constants and time constants of overall rotational diffusion is essential. When the transition between different bending states of standard DNA is slow compared to overall rotational diffusion, the transients upon reversal of the field vector cannot be distinguished from those for DNA with static curvature. Finally, all intermediate cases with different ratios of the time constants are possible. The analysis of these complex cases is not simple. A useful approach should be simulation of data by Brownian dynamics for comparison with experimental data.

Bending Dynamics of DNA. DNA bending time constants have been obtained from measurements of the electric dichroism at high field strengths. ${ }^{25,40}$ Pulses of high field strengths induce stretching of bent conformations to more straight ones. After pulse termination the reverse reaction corresponding to bending is indicated by a fast component in the dichroism decay. For example, a fragment with 179 bp shows a bending time constant of $\sim 180 \mathrm{~ns}$ at $20^{\circ} \mathrm{C}$, which is much below the time constant of overall rotational diffusion $(\sim 3 \mu \mathrm{s})$. The bending time constants observed under these conditions strongly increase with increasing chain lengths. In all these cases the observed bending times indicate a relatively fast bending reaction.
A different conclusion was suggested by the observation of a positive stationary dichroism and quasi-permanent dipole moments for various DNA fragments ${ }^{32}$ in the range of chain lengths from 399 to $859 \mathrm{bp}$. Most of these fragments did not show an unusual gel migration and, thus, were considered to be normal DNA. As discussed in the previous section, the existence of a large dip in the transients upon pulse reversal was taken as evidence for a low internal mobility: the data are consistent with simulations on rigid wormlike chains. ${ }^{32}$

This conclusion may not be justified if the fragments used in these experiments are not really straight DNA, but contain elements of curvature. Predictions from sequences based on available models rarely provide completely straight helix trajectories and usually show at least some degree of curvature.

Finally, the difference of time constants after pulses of low and high electric field strengths, observed in the present investigation, indicates a relatively slow transition between different bending states. If the internal equilibration would be fast compared to rotational diffusion, the time constant of overall rotational diffusion should be independent of the electric field strength used for alignment.

In summary, there are different indications on the rate of DNA bending. This suggests the possibility that there are different modes of DNA bending. Small deviations from equilibrium conformations may relax quickly, such that the time constants are determined by hydrodynamic friction. Larger deviations involving stacking rearrangements are determined by stacking rates, which are known to be relatively low, ${ }^{41,42}$ at least in some cases. More investigations on the bending dynamics of DNA are required for final conclusions.

Hydrodynamic Dimensions and Their Quantitative Assignment. The time constants, in particular those measured at high salt concentrations, confirm the expectation that the overall hydrodynamic dimensions of curved DNA are smaller than those of standard DNA. The reduction of this difference at low salt concentrations is partly due to electrostatic repulsion between the phosphate charges, driving the polymer chain into a more extended conformation. Another contribution may result from dipolar stretching of curved DNA, which is extreme at low salt concentrations. Dipolar stretching is reduced to a minimum at $100 \mathrm{mM}$ salt, because the polarizability is decreased by about 2 orders of magnitude under these conditions.

The interpretation of the time constants would be relatively easy, if the basis were as simple as believed by many authors until recently. Usually it has been assumed that the internal mobility of DNA chains is high, ${ }^{43,44}$ such that conversion between different conformations of the chain is fast compared to overall rotational diffusion. As discussed in the previous section, there may be more than a single mode of DNA bending in different time scales. A clear effect of the internal mobility on the electrooptical time constants of wormlike chains has been demonstrated. ${ }^{45}$

Simulations of the electrooptical transients for a distribution of different conformers should take care of various other effects. For example, the dependence of the polarizability on the shape of the conformer should be included. It must be expected that extended conformers have a higher polarizability in the direction of their maximal extension than more compact conformers. Thus, at low electric field strengths extended conformers will be reflected in the decay curves preferentially, whereas more compact ones will contribute more clearly at higher field strengths. This expectation is consistent with the present observations. 
Recent calculations on curved DNA fragments demonstrated that hydrodynamic coupling of translational and rotational diffusion strongly affects the electrooptical data of simple arc models. ${ }^{30}$ Although the coupling effect is expected to be less extensive in the case of wormlike chain conformers, it cannot be neglected. Thus, the required model calculations are more involved than those presented previously. Such calculations (in progress) together with the present electrooptical data will serve to evaluate the degree of curvature and to assess the validity of currently available models for DNA curvature. These calculations will not only use the information contained in the time constant of overall rotational diffusion but also include the special form of the transients and the additional relaxation process. Thus, the new data should enable a more accurate assignment of DNA curvature than possible previously.

\section{Summary}

Using an improved birefringence technique, it has been possible for the first time to measure electrooptical data of curved DNA at low electric field strengths over a wide range of ion concentrations from 0.6 to $100 \mathrm{mM} \mathrm{Na}^{+}$. The time constants demonstrate a clear increase of the curvature with increasing salt concentration as should be expected from electrostatic repulsion. The appearance of a positive stationary birefringence together with transients characteristic of a permanent dipole at high salt concentration are consistent with preferential bending in a given direction as implied by standard curvature models. In the case of high internal bending dynamics, electrooptical transients upon reversal of the field vector can be used to distinguish static from dynamic persistence. This not possible, however, when bending transitions are slow compared to overall rotational diffusion.

Acknowledgment. The author is indebted to C. Reichardt and Prof. D. Schwarzer for their generosity of granting access to their laser, when the author's own laser could not be used anymore. The technical assistance of Jürgen Wawrzinek is acknowledged. The facilities of the Gesellschaft für wissenschaftliche Datenverarbeitung $\mathrm{mbH}$ Göttingen were used for computations.

\section{References and Notes}

(1) Trifonov, E. N. CRC Crit. Rev. Biochem. 1985, 19, 89.

(2) Hagerman, P. J. Аnnu. Rev. Biochem. 1990, 59, 755.

(3) Harrington, R. E. Electrophoresis 1993, 14, 732.

(4) Koo, H. S.; Drak, J.; Rice, J. A.; Crothers, D. M. Biochemistry 1990, 29, 4227

(5) Zhang, Y. L.; Crothers, D. M. Biophys. J. 2003, 84, 136.

(6) MacDonald, D.; Herbert, K.; Zhang, X. L.; Polgruto, T.; Lu, P. J. Mol. Biol. 2001, 306, 1081 8173.

(7) Toth, K.; Sauermann, V.; Langowski, J. Biochemistry 1998, 37,

(8) Tchernaenko, V.; Halvorson, H. R.; Lutter, L. C. J. Mol. Biol. 2004, 341,55

(9) Shpigelman, E. S.; Trifonov, E. N.; Bolshoy, A. Comput. Appl. Biosci. 1993, 9, 435 .

(10) Vlahovicek, K.; Kajan, L.; Pongor, S. Nucleic Acids Res. 2003, 31,3686 .

(11) Anselmi, C.; De Santis, R.; Paparcone, R.; Savino, M.; Scipioni, A. Biophys. Chem. 2002, 95, 23.

(12) Fredericq, E.; Houssier, C. Electric dichroism and electric birefringence; Clarendon Press: Oxford, 1973.

(13) Porschke, D.; Antosiewicz, J. M. Quantitative Molecular ElectroOptics: Macromolecular Structures and Their Dynamics in Solution. In Molecular and colloidal electro-optics; Stoylov, S. P., Stoimenova, M. V., Eds.; CRC: Boca Raton, FL, 2007.

(14) Porschke, D.; Geisler, N.; Hillen, W. Nucleic Acids Res. 1982, 10 , 3791.

(15) Porschke, D. Biophys. J. 1998, 75, 528.

(16) Marini, J. C.; Levene, S. D.; Crothers, D. M.; Englund, P. T. Proc Natl. Acad. Sci. U.S.A. 1982, 79, 7664.

(17) Hagerman, P. J. Proc. Natl. Acad. Sci. U.S.A. 1984, 81, 4632.

(18) Levene, S. D.; Wu, H. M.; Crothers, D. M. Biochemistry 1986 25,3988 .

(19) Diekmann, S.; Porschke, D. Biophys. Chem. 1987, 26, 207.

(20) Chan, S. S.; Breslauer, K. J.; Austin, R. H.; Hogan, M. E. Biochemistry 1993, 32, 11776.

(21) Lu, Y. J.; Weers, B. D.; Stellwagen, N. C. Biophys. J. 2005, 88, 1191.

(22) Porschke, D.; Obst, A. Rev. Sci. Instrum. 1991, 62, 818.

(23) Porschke, D. Biophys. Chem. 1991, 40, 169.

(24) Porschke, D.; Schmidt, E. R.; Hankeln, T.; Nolte, G.; Antosiewicz, J. Biophys. Chem. 1993, 47, 179.

(25) Diekmann, S.; Hillen, W.; Morgeneyer, B.; Wells, R. D.; Porschke,

D. Biophys. Chem. 1982, 15, 263.

(26) Porschke, D.; Jung, M. J. Biomol. Struct. Dyn. 1985, 2, 1173.

(27) Tinoco, I.; Yamaoka, K. J. Phys. Chem. 1959, 63, 423.

(28) Hagerman, P. J. Аnnu. Rev. Biophys. Biophys. Chem. 1988, 17, 265.

(29) Antosiewicz, J.; Porschke, D. Biophys. Chem. 1989, 33, 19.

(30) Porschke, D.; Antosiewicz, J. M. J. Phys. Chem. B 2005, 109, 1034.

(31) Porschke, D. Colloids Surf., B 2007, 56, 44.

(32) Porschke, D. Biophys. Chem. 1994, 49, 127

(33) Yamaoka, K. Colloids Surf., A: Phys. Chem. Eng. Aspects 2002, 209, 243

(34) Porschke, D. Biophys. Chem. 1985, 22, 237.

(35) Diebler, H.; Eigen, M.; Ilgenfritz, G.; Maass, G.; Winkler, R. Pure Appl. Chem. 1969, $20,93$.

(36) Antosiewicz, J.; Porschke, D. J. Phys. Chem. B 1997, 101, 4478

(37) Porschke, D. Biophys. Chem. 1987, 28, 137.

(38) Porschke, D.; Tovar, K.; Antosiewicz, J. Biochemistry 1988, 27, 4674.

(39) Trifonov, E. N.; Tan, R. K. Z.; Harvey, S. C. Static persistence length of DNA. In Structure \& Expression; Olson, W. K., Sarma, M. H. Sarma, R. H., Sundaralingam, M., Eds.; Adenine Press: Schenectady, NY, 1987; Vol. 3: DNA Bending and Curvature; p 243.

(40) Porschke, D. J. Biomol. Struct. Dyn. 1986, 4, 373.

(41) Porschke, D. Biochemistry 1976, 15, 1495.

(42) Porschke, D. Biopolymers 1978, 17, 315.

(43) Hagerman, P. J.; Zimm, B. H. Biopolymers 1981, 20, 1481.

(44) Vacano, E.; Hagerman, P. J. Biophys. J. 1997, 73, 306. 6500 